\title{
Design of the SABAT system for underwater detection of dangerous substances*
}

\author{
M. Silarski ${ }^{a}, \mathrm{D} \cdot \operatorname{HuniK}^{a}, \mathrm{M} \cdot \mathrm{Smolis}^{a}, \mathrm{~S} \cdot \mathrm{Tadeja}^{a}, \mathrm{P} \mathrm{Moskal}^{a}$ \\ ${ }^{a}$ Institute of Physics, Jagiellonian University \\ Łojasiewicza 11, 30-348 Kraków, Poland
}

\begin{abstract}
We present status of simulations used to design a novel device for the detection of hazardous substances in the aquatic environment using neutron activation. Unlike the other considered methods based on this technique we propose to use guides for neutron and gamma quanta which speeds up and simplifies identification. First preliminary results show that both the neutron guide and the $\gamma$ ray guide increase the performance of underwater threats detection.
\end{abstract}

PACS numbers: P82.80.Jp, 89.20.Dd

\section{Introduction}

Growing risk of terrorism demands a constant development of new techniques for hazardous substances detection. One of the best methods for homeland security are based on the Neutron Activation Analysis (NAA) providing non-intrusive detection of explosives or drugs. The principle of the method is to use a fast neutron beam produced using sealed $\mathrm{D}+\mathrm{T}$ generator to excite nuclei of investigated substance and detect characteristic gamma quanta produced in de-excitation of the nuclei. This allows one to identify the stoichiometry of the substance and determine if it is dangereus [1, 2]. So far there are several devices based on Neutron Activation Analysis designed for ground homeland security which were introduced in the USA by Science Applications International Corporation [3] and CALSEC [4], and in Europe in Sodern [6], EURITRACK [5] and SWAN [7] projects. These devices are used in the homeland security or contraband detection on the land. However, in an aquatic environment one encounters serious problems due to strong attenuation of neutrons in water. Moreover, as in the case

* Presented at the Jagiellonian Symposium of Fundamental and Applied Subatomic Physics, Kraków, 11.06.2015 
of ground detectors, an isotropic generation of neutrons induces a large environmental background. This noise can be significantly reduced by the requirement of the coincident detection of the alpha particles which are produced together with neutrons [8, 9]. The attenuation of neutrons can be compensated by reducing the distance between generator and examined item [10]. There are also solutions based on low energy neutrons which are moderated in water before reaching the tested object. The detector is then counting the gamma quanta from thermal neutron capture and secondary neutrons originating from the irradiated object. The identification is done by searching for anomalies in the observed spectra of gamma quanta and neutrons [11]. However, these methods do not allow to detect explosives buried deeper in the bottom of the sea and strong attenuation of neutrons and gamma quanta significantly increases the exposure time.

An alternative solution of a detector which uses NAA technique and special guides for neutrons and emitted gamma rays was proposed within the SABAT project [12]. The device allows for detection of dangerous substances hidden deep in the bottom of the sea with significantly reduced background and provides determination of the density distribution of the dangerous substance in the tested object [11]. In this article we present status of the design of the SABAT detector based on Monte Carlo simulations, focusing on the impact of the usage of neutron and gamma quanta guides.

\section{Status of the simulation}

In order to optimize the dimensions and relative positions of detectors and guides we have developed dedicated open source software package written in the $\mathrm{C}++$ programming language [11]. This simulation tool is using novel methods of geometry definition and particle tracking and the Open MPI library supporting parallel computing [13]. To define the geometry of simulated objects we use a polygon mesh, which is in the simplest form a collection of vertices and edges that are used to define a surface of the polyhedral 3D object. In our case the faces consist of triangles. Trajectory of a particle traveling in a scene consisting of such defined objects is simulated with so-called ray tracing technique. To determine if a particle entered given object the Möller-Trumbore method was implemented which searches for intersection of the particle trajectory with the object surface (triangle mesh). The total cross-sections and angular distributions of neutrons and $\gamma$ rays interaction with selected nuclei were parametrized as a function of energy using data from the Evaluated Nuclear Data File (ENDF) database [14], while gamma quanta energies were taken from the Evaluated Nuclear Structure Data Files (ENSDF) [15]. As a starting point for design of the device for underwater threats detection we have defined a simple setup 
a)
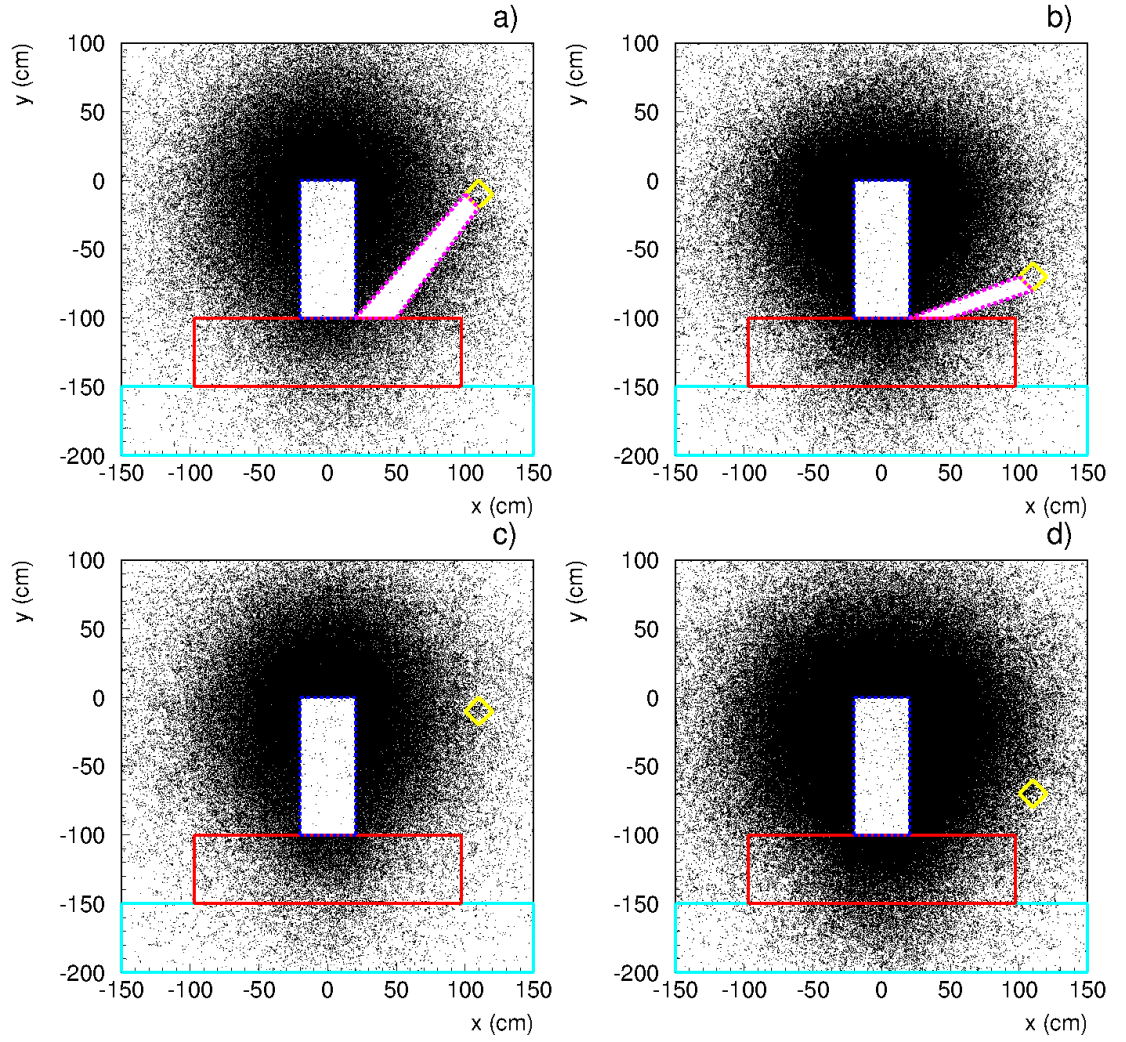

Fig. 1. Distribution of $\gamma$ quanta interaction points in $x-y$ views for two positions of the detector with ( $\mathrm{a}$ and $\mathrm{b}$ ) and without gamma quanta guide ( $\mathrm{c}$ and $\mathrm{d}$ ). The point-like source of neutrons is located at the origin of the reference frame. The neutron guide is presented as the blue dashed rectangle aligned along the y axis. The container with mustard gas is a red cuboid aligned horizontally which lies at the bottom of the sea marked in cyan. The Ge detector (in yellow) is connected to the gamma rays guide (dashed magenta polyhedron). For better visibility of the influence of guides plots are made only for $-10 \leq \mathrm{z} \leq 10 \mathrm{~cm}$.

with point-like source generating uniformly in space $14.1 \mathrm{MeV}$ neutrons in the neutron- $\alpha$ center of mass frame. As a first step, to study the influence on the measurement of gamma quanta guide and the relative position of generator and detector we have simulated $1.4 \cdot 10^{7}$ events with two different detector locations with and without gamma rays guide. The scheme of the simulated setups with superimposed $\gamma$ quanta interaction points are shown in Fig. 1. The interrogated object with dimensions $194 \times 50 \times 50 \mathrm{~cm}^{3}$ lies at the bottom of a sea and contains mustard gas $\left(\mathrm{C}_{4} \mathrm{H}_{8} \mathrm{Cl}_{2} \mathrm{~S}\right)$. The neutron guide is a cuboid with dimensions $40 \times 100 \times 40 \mathrm{~cm}^{3}$, while gamma rays 


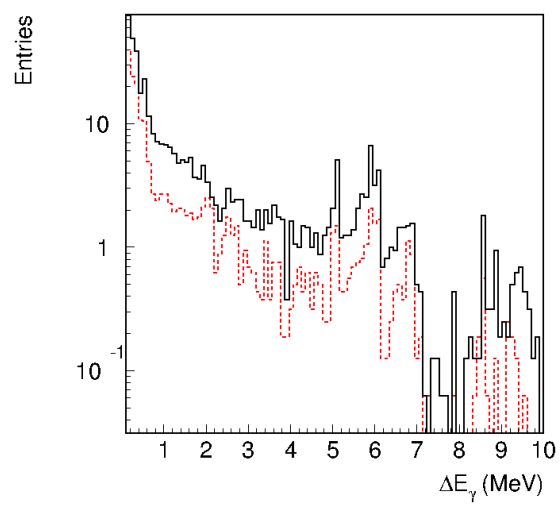

Fig. 2. Simulated energy spectra as would be measured with the germanium detector. Simulations were performed for the setups presented in Fig. 1b (solid histogram) and in Fig. 11d (dashed histogram). The small signal from the mustard gas can be seen at about $9.6 \mathrm{MeV}$ and $4.4 \mathrm{MeV}$ (carbon), 2.2 MeV (sulfur) and around $6.5 \mathrm{MeV}$ (chlorine). The huge background is mainly due to the gamma quanta from neutron-oxygen reactions. The energy of these quanta is smeared due to the scattering in the water. The number of entries is normalized to the generated statistics.

Table 1. Fractional composition of sources of gamma quanta registered by the detector (in \%) for all the simulated cases shown in Fig. 1. The last column shows the overall fraction of gamma quanta reaching the detector and originating from the mustard gas.

\begin{tabular}{|c|c|c|c|c|c|c|c|c|c|c|}
\hline Setup & $\mathrm{H}$ & $\mathrm{C}$ & $\mathrm{N}$ & $\mathrm{O}$ & $\mathrm{Na}$ & $\mathrm{Si}$ & $\mathrm{S}$ & $\mathrm{Cl}$ & $\mathrm{n}$ & $\begin{array}{c}\text { Mustard } \\
\text { Gas }\end{array}$ \\
\hline $\mathrm{a}$ & 0 & 0.77 & 0 & 85.17 & 0.24 & 0 & 0 & 0.26 & 13.56 & 1.02 \\
\hline $\mathrm{b}$ & 0.53 & 1.68 & 0.10 & 76.50 & 0.38 & 0.08 & 0.63 & 1.78 & 18.32 & 3.30 \\
\hline $\mathrm{c}$ & 0.59 & 0 & 0 & 81.71 & 0.88 & 0 & 0 & 0.89 & 15.93 & 0 \\
\hline $\mathrm{d}$ & 7.88 & 0.59 & 0.05 & 77.04 & 0.69 & 0.24 & 0.10 & 6.95 & 6.46 & 2.25 \\
\hline
\end{tabular}

guides is represented by polyhedron with $15 \times 20 \mathrm{~cm}^{2}$ and $30 \mathrm{x} 30 \mathrm{~cm}^{2}$ bases. They are both filled with air under normal conditions. As a detector we have simulated $15 \times 15 \times 20 \mathrm{~cm}^{3}$ Ge crystal measuring the energy of gamma rays with $6 \%$ resolution (FWHM). The detector surface with dimensions of $15 \times 20 \mathrm{~cm}^{2}$ is adjusted to the shape of the gamma quanta guide. The exemplary energy spectra measured by the germanium detector for one detector position are shown in Fig. 2, As one can see in Tab. 1. among all of the gamma quanta reaching the detector about $80 \%$ originate from oxygen in the water independently of the detector position or presence of the guide. The second relevant source of background constitute neutrons 
which scatter in water and reach the detector. The amount of registered gamma quanta which were created in the container with mustard gas is quite small but the advantage of using guide is visible. The mustard gas is composed of carbon, hydrogen, chlorine and sulfur. However, Fig. 2 indicates that the $4.4 \mathrm{MeV}$ carbon line is overwhelmed by background while we see clearly gamma quanta from carbon with energy of about 9.6 MeV. In case of chlorine and sulfur (lines of $6.5 \mathrm{MeV}$ and $2.2 \mathrm{MeV}$, respectively) the oxygen background has to be significantly reduced to allow one to identify these elements more clearly. This can be done requiring of the coincident detection of the $\alpha$ particle generated together with neutron or by covering all the detector faces not connected to the gamma quanta guide with thick layer of absorber. Identification of sulfur and chlorine is difficult due to neutron capture on hydrogen giving lines of very close energy and chlorine content in the sea water 11. However, using the gamma quanta guide one can detect $\gamma$ rays from these two elements not affected by scattering in the water which gives a great advantage in the identification.

\section{Conclusions and outlook}

In the framework of the SABAT project we have been developing simulation package based on novel methods of geometry definition and particle tracking. Although we are still in a very early stage of development the first results indicate that indeed, both the neutron guide and $\gamma$ ray guide will increase the performance of underwater threats detection with fast neu-

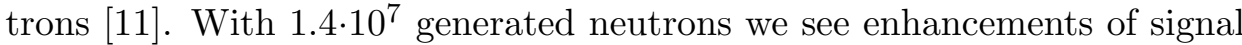
events in the detector together with huge background originating mostly from oxygen, as it was expected. This shows clearly that all the faces of the detector not connected to the guide should be covered with thick layer of material absorbing $\gamma$ quanta and neutrons which constitute another big source of background. This noise can be also significantly reduced by the requirement of the coincident detection of the $\alpha$ particle generated together with neutron, which allows for the neutron tagging [11]. As one of the possible way of the background suppression via time-of-flight method we consider an application of plastic scintillators in order to take advantage of their excellent timing properties, as recently demonstrated in case of low energy gamma quanta [16, 17, 18, 19]. The $\alpha$ particle detection and time measurement will be included as the next step in the development of the simulations. This will allow for a final design of the device for underwater threats detection with fast neutrons, which requires much bigger statistics generated. 


\section{REFERENCES}

[1] P. Moskal, Annales UMCS, Physica 66, 71 (2012)

[2] M. Silarski, Acta Phys. Pol. B Suppl. 6, 1061 (2013)

[3] http://phoenixnuclearlabs.com/solution/explosives-detection/

[4] B. C. Maglich, AIP Conf. Proc. 796, 431 (2005); http://www.calseco.com/

[5] http://www.euritrack.org/

[6] http://www.sodern.com/

[7] Ł. Kaźmierczak et. al., Acta Phys. Polon. A 127, 1540 (2015); http://aid.ncbj.gov.pl/index.php/en/products/swan

[8] D. Sudac, S. Pesente, G. Nebbia, G. Viesti, V. Valkovic, Nucl. Instrum. Meth. B 261, 321 (2007)

[9] S. Pesente, PhD Thesis, University of Padova (2003)

[10] C. Eleon, B. Perot, C. Carasco, D. Sudac, J. Obhodas, V. Valkovic, Nucl. Instrum. Meth. A 629, 220 (2011)

[11] M. Silarski, D. Hunik, P. Moskal, M. Smolis, S. Tadeja, Acta Phys. Polon. A 127, $1543(2015)$

[12] M. Silarski, P. Moskal, International patent application No. PCT/PL2015/ 050021

[13] M.J. Quinn, Parallel Programming in C with MPI and OpenMP, McGraw-Hill, 2004

[14] M.B. Chadwick et al., Nucl. Data Sheets 112(12), 2887 (2011).

[15] M. R. Bhat, Nuclear Data for Science and Technology (revised as of April 2014) edited by S. M. Qaim (Springer-Verlag, Berlin, Germany, 1992)

[16] L. Raczyński et al., Nucl. Instrum. Meth. A 764, 186 (2014)

[17] P. Moskal et al., Nucl. Instrum. Meth. A 764, 317 (2014)

[18] L. Raczyński et al., Nucl. Instrum. Meth. A 786, 105 (2015)

[19] P. Moskal et al., Nucl. Instrum. Meth. A 775, 54 (2015) 